\title{
IUFOST2006/1092
}

\section{Consumer Targeted Product Quality: how to keep your consumers happy}

\author{
M. Everitt \\ Sensory Dimensions, Science \& Technology Centre, Earley Gate, RG6 6BZ Reading, United Kingdom \\ margaret@sensorydimensions.com
}

\begin{abstract}
Since man first started trading on a commercial scale, some type of "specification" has been required against which the quality (expected or implied) of the goods can be measured. In today's global food markets these product specifications have become an integral part of a company's quality assurance programme. Specific information relating to legal aspects, packaging, microbial safety, ingredients, weight, nutritional content, etc are usually detailed at length yet the sensory component in many cases remains poorly defined and open to individual interpretation.

Why is this? There are many common challenges and issues that face the implementation of a practical and reliable sensory quality programme, for manufacturers and suppliers across the food industry. Identifying the sensory characteristics most important to consumers' acceptance of a product is the primary challenge. Once defined this then enables the sensory quality targets for key characteristics to be more objectively set. The importance of defects, both type and quantity, are also key in specifying the quality that will be acceptable to the marketplace.

This presentation will discuss these challenges and look at how to define and implement an effective sensory quality system to help ensure that it is your consumers' that keep coming back, not your product.
\end{abstract}

